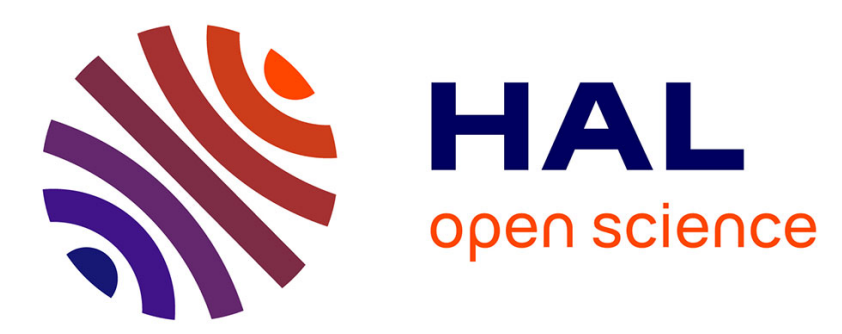

\title{
Interconnection of asynchronous Boolean networks, asymptotic and transient dynamics
}

Laurent Tournier, Madalena Chaves

\section{To cite this version:}

Laurent Tournier, Madalena Chaves. Interconnection of asynchronous Boolean networks, asymptotic and transient dynamics. Automatica, 2013, 49 (4), pp.884-893. 10.1016/j.automatica.2013.01.015 . hal-00848450

\section{HAL Id: hal-00848450 \\ https://inria.hal.science/hal-00848450}

Submitted on 26 Jul 2013

HAL is a multi-disciplinary open access archive for the deposit and dissemination of scientific research documents, whether they are published or not. The documents may come from teaching and research institutions in France or abroad, or from public or private research centers.
L'archive ouverte pluridisciplinaire $\mathbf{H A L}$, est destinée au dépôt et à la diffusion de documents scientifiques de niveau recherche, publiés ou non, émanant des établissements d'enseignement et de recherche français ou étrangers, des laboratoires publics ou privés. 


\title{
Interconnection of asynchronous Boolean networks, asymptotic and transient dynamics
}

\author{
Laurent Tournier* Madalena Chaves ${ }^{\dagger}$
}

\begin{abstract}
The dynamics of the interconnection of two Boolean networks is analyzed directly from the properties of the two individual modules. Motivated by biological systems where multiple timescales are present, we consider asynchronous Boolean networks, whose dynamics can be described by non-deterministic transition graphs. Two new objects are introduced, the asymptotic and the cross- graphs, constructed from the strongly connected components of the modules' transition graphs. It is then proved that the asymptotic graph actually recovers the attractors of the interconnected system, while reducing overall computational cost. Illustrated by various biological applications, this method is applied to analyze a composition of several well known modules (multicellular modeling), or to analyze a high dimensional model through its decomposition into smaller input/output subnetworks (model reduction).
\end{abstract}

\section{Introduction}

Analysis of large networks is an increasingly recurrent problem in many fields, notably in the biological sciences. Large networks are often obtained in a modular way, by first developing separate input/output modules for several smaller subsystems [15] whose dynamical behavior can, in general, be completely characterized. It is, however, much more difficult to predict the global behavior of the full interconnected network. In this paper, a solution to this problem is proposed by studying the dynamical properties of the interconnection of two (or more) modules directly from the properties of the individual input/output modules. System interconnection is a classical concept in automatic control, and it has been used before to predict the dynamics of composed systems. For discrete systems, the graph theoretic representation has led to various computer science approaches to analyze reachability and other properties of an interconnected system [13]. For continuous systems, the existing approaches are valid only under particular assumptions on the modules: in [3], the equilibria of an interconnection of two monotone control systems was analyzed based on the existence of static input-output characteristics. Here, we will focus on asynchronous Boolean networks and obtain a general theoretical result which is valid for any interconnection. Boolean networks are an efficient framework for representing large complex networks of interactions, providing good qualitative description and predictive power [4] even when not much data is available. Relevant examples include models of embryogenesis of the fruit fly [2, 7]; yeast or mammalian cell cycles [17, 12]; leukemia [26]; and apoptosis [5, 21]. In contrast to synchronous networks, asynchronous networks have nondeterministic dynamics, and capture more faithfully the dynamics of a system with distinct time-scales, such as cellular regulatory networks [7].

The state space and dynamics of a Boolean network with $n$ variables can be represented by a directed graph of dimension $2^{n}$. In principle, this graph can be fully analyzed using graph theoretical concepts and tools [23], and some classical algorithms [11], to describe transient and asymptotic behavior. The drawback is that such techniques become computationally prohibitive when the dimension increases, and hence the importance of using the knowledge of each separate module.

Two new objects are introduced, the asymptotic graph and the cross-graph, that allow to recover the attractors of the interconnected system. The cross-graph provides a complete theoretical characterization of the interconnected system, but is nonpractical for computational purposes, while the asymptotic graph provides only a sufficient condition, but can be easily applied and generally leads to a major decrease of the computational cost.

\footnotetext{
${ }^{*}$ Corresponding author. INRA - unit MIG, UR 1077, Domaine de Vilvert, F-78350 Jouy-en-Josas, France, laurent.tournierejouy.inra.fr

†BIOCORE, INRIA, 2004 Route des Lucioles, BP 93, 06902 Sophia Antipolis, France,madalena . chaves@inria.fr
} 
It can be used in two ways: first, given two well known modules, one can rapidly retrieve the asymptotic dynamics of the network arising from the composition of the two modules. This is useful for instance for the analysis of multicellular networks, such as the segment polarity network in drosophila embryogenesis. Alternatively, a high dimensional network can be decomposed into smaller Boolean input/output modules, thus generating a novel model reduction method.

To present our results, some basic concepts on directed graphs are first quickly summarized (Section 2), as well as the definitions of asynchronous Boolean input/output modules and their interconnections (Section 3). The asymptotic and cross- graphs, and their corresponding results (Theorems 1 and 2), are introduced in Section 4. The asymptotic graph has been previously studied in [8] but here an alternative proof will be given, based on the one-to-one correspondence obtained for the cross-graph (Section 5). Finally, Sections 6 and 7 present the application of the method to multicellular modeling and model reduction. Two biological illustrations are given, in which it was possible to recover all known attractors and establish that no complex attractors can exist - a result which was not possible to achieve with classical, simulation-based techniques.

\section{Directed graphs: definitions and notation}

We begin by summarizing some notation and basic definitions on graphs that will be used throughout this paper. For a detailed introduction to graph theory, the reader is referred to general textbooks such as $[11,14]$. Let $G=(V, E)$ be a directed graph: $V$ is the (finite) set of vertices and $E \subset V \times V$ is the set of (directed) edges. All edges are supposed simple. Let $x$ and $y$ designate two vertices of $V$, and define:

- $x \rightarrow_{G} y$ means that there is an edge from $x$ to $y$ in $G$, i.e. $(x, y) \in E$. Then $y$ is called a successor of $x$.

- $x \triangleright_{G} y$ means that there exists a path from $x$ to $y$ in $G$, i.e. there exist $k \geq 0$ vertices $x_{1}, \ldots, x_{k}$ such that $x \rightarrow_{G} x_{1} \rightarrow_{G} x_{2} \rightarrow_{G} \ldots \rightarrow_{G} x_{k} \rightarrow_{G} y$. Then $y$ is called a descendant ${ }^{1}$ of $x$.

- $x \sim_{G} y$ means that $x$ and $y$ are mutually reachable from each other, i.e. $x \triangleright_{G} y$ and $y \triangleright_{G} x$.

The binary relation $\sim_{G}$ is an equivalence over $V$; an equivalence class of $\sim_{G}$ is called a strongly connected component (SCC) of $G$. By definition, the SCCs of $G$ define a partition of the set of vertices $V$. A SCC will be called terminal if it has no outgoing edges (in any graph, at least one SCC is terminal). A set of vertices which are mutually reachable two by two will be said strongly connected (a strongly connected set is necessarily included in a SCC). For any vertex $x \in V$, the reachable set $\mathcal{R}(x)$ of $x$ is the set of all the descendants of $x$. If $Q$ designates the SCC containing $x$ (it is unique by definition), any vertex $y \in Q$ has the same reachable set as $x$. Finally, a non-empty set of vertices $U \subset V$ will be called an invariant set of $G$ if $\mathcal{R}(x) \subset U$ for any $x \in U$. The following lemma is a direct consequence of these definitions, and will be used later on. Its proof is straightforward.

Lemma $1 G=(V, E)$ is a directed graph. Any invariant set $U \subset V$ contains at least one terminal SCC of $G$.

For any $x \in V$, the reachable set of $x$ is clearly an invariant of $G$. In other words, Lemma 1 implies that any vertex $x$ leads to at least one terminal SCC of $G$. The algorithmic search for SCCs of a graph $G=(V, E)$ is well known (see e.g. [11]) and can be done linearly in $|V|+|E|$. Once the SCCs are identified, one can straightforwardly build a SCC-graph by putting an edge from a SCC $Q$ to a SCC $Q^{\prime}$ iff there is $x \in Q$ and $y \in Q^{\prime}$ such that $x \rightarrow_{G} y$. By construction, this SCC-graph is acyclic and therefore can be topologically sorted [11]. This sorting provides a hierarchical organization of the SCCs of $G$, and hence of the set of vertices $V$. The resulting treelike structure allows the direct visualization of key elements of $G$. For instance, the reachable set of a SCC $Q$ is constituted by the subtree stemming from $Q$, and the terminal SCCs of $G$ are the leaf nodes. Similarly, invariants of $G$ are easy to detect by walking the tree from any set of SCCs down to the leaf nodes. These are classical results (SCC decomposition, topological sort) that can be implemented in matlab. They have been applied to all graphs presented in this paper.

Finally, we will use classical notations for Boolean operators. If $x, y \in\{0,1\}$, the conjunction of $x$ and $y$ ( $x$ and $y$ ) will be denoted by the product $x y$, the disjunction of $x$ and $y(x$ or $y$ ) will be denoted by $x \vee y$, and the negation of $x$ will be denoted by $\bar{x}$.

\footnotetext{
${ }^{1}$ Thus, every $x \in V$ is always a descendant of itself.
} 


\section{Interconnection of Boolean modules}

\subsection{Input-output asynchronous Boolean networks}

We now briefly recapitulate the definition of an asynchronous Boolean network (see for instance [5, 23]) and generalize it to include inputs and outputs. An input-output asynchronous Boolean network (IO ABN) $\Sigma^{A}$, of dimension $n_{A}>0$, is characterized by the following five components: its state space $\Omega^{A}=\{0,1\}^{n_{A}}$, its input and output sets $\mathcal{U}^{A}=\{0,1\}^{p_{A}}$ and $\mathcal{H}^{A}=\{0,1\}^{q_{A}}\left(p_{A}, q_{A} \geq 0\right)$, a transition function $f^{A}: \Omega^{A} \times \mathcal{U}^{A} \rightarrow \Omega^{A}$ and an output function $h^{A}: \Omega^{A} \rightarrow \mathcal{H}^{A}$. The state variable is denoted by $a=\left(a_{1}, \ldots, a_{n_{A}}\right) \in \Omega^{A}$ and the input variable is denoted by $u \in \mathcal{U}^{A}$.

It is assumed that the state of the network can be updated at a discrete sequence of time instants, and that the state at the next instant depends only on the input and state of the system at the current instant. Two general strategies exist, leading to very different dynamics. The synchronous strategy, where all variables are updated simultaneously at each time, generates deterministic dynamics (see e.g. $[4,10,17])$. This strategy is not always appropriate from a modeling point of view, especially if the network involves processes taking place at different time scales, which is often the case in biological systems [19]. Therefore, we adopt the more general asynchronous strategy, where only one variable is updated at each time. More precisely, consider a state $a \in \Omega^{A}$ and an input $u \in \mathcal{U}^{A}$, then for any index $l$ such that $a_{l} \neq f_{l}^{A}(a, u)$, the state

$$
\widetilde{a}^{l}:=\left(a_{1}, \ldots, \overline{a_{l}}, \ldots, a_{n_{A}}\right)
$$

is a possible successor of $a$. This strategy implies that each state may have several (up to $n_{A}$ ) distinct successors, and is more reliable for the analysis of global dynamical properties, regardless of the relative speeds of the processes involved. Note that contrary to other asynchronous schemes $[6,7]$, no predefined updating order is imposed (whether it be fixed or random), allowing to model systems where timescales are completely unknown. Therefore, all results presented in this paper are independent of the updating order and can be generalized to more specific asynchronous strategies ${ }^{2}$.

The analysis of an ABN without inputs or outputs can be found for instance in $[5,23]$. For IO ABN, the relevant objects are defined as follows.

Definition 1 The asynchronous transition graph of network $\Sigma^{A}$ under fixed input $u \in \mathcal{U}^{A}$ is denoted by $G^{A, u}$. Its set of vertices is the state space $\Omega^{A}$, and its edges are determined by the following rule. For any state $a \in \Omega^{A}$ :

i) if $a \neq f^{A}(a, u)$, then $a \rightarrow{ }_{G}^{A}, u \widetilde{a}^{l}$ (see Eq. (1)), for all indices $l$ such that $a_{l} \neq f_{l}^{A}(a, u)$,

ii) if $a=f^{A}(a, u)$, then $a \rightarrow{ }_{G}^{A, u} a$ ( $a$ is a steady-state of the network).

With this definition, every state $a \in \Omega^{A}$ has at least one successor. The asynchronous dynamics of $\Sigma^{A}$ is given by all the transition graphs $G^{A, u}, u$ lying in $\mathcal{U}^{A}$. There are $2^{p_{A}}$ such graphs, each of size $2^{n_{A}}$. The SCCs of a transition graph are next characterized according to their output.

Definition 2 Let $A_{u}^{1}, \ldots, A_{u}^{N_{u}}$ denote the SCCs of $G^{A, u}\left(N_{u} \geq 1\right)$. Given a SCC $A_{u}^{i}$ and an output $\alpha \in \mathcal{H}^{A}$, the semi-SCC of $A_{u}^{i}$ of output $\alpha$ is the subset: $A_{u \alpha}^{i}:=\left\{a \in A_{u}^{i}, h^{A}(a)=\alpha\right\}$. If $A_{u}^{i}$ is terminal it will be called attractor of the network under input $u$, and its semi-SCCs will be called semi-attractors.

A direct consequence is that $\cup_{\alpha \in \mathcal{H}^{A}} A_{u \alpha}^{i}=A_{u}^{i}$ (the union is disjoint). For some $\alpha$, the set $A_{u \alpha}^{i}$ can be empty. However, for $u$ and $i$ fixed, there always exists at least one $\alpha$ for which $A_{u \alpha}^{i} \neq \varnothing$. By convention, throughout the paper we will consider only non-empty semi-SCCs (empty ones will simply be omitted).

Example 1 In order to illustrate these definitions, consider the single input - single output (SISO) bidimensional network, composed of a negative feedback loop:

$$
u \cdots \cdots \cdots, a_{1}>a_{2} \cdots \cdots \cdots, h^{A}
$$

The state space is $\Omega^{A}=\{0,1\}^{2}$, the input and output spaces are $\mathcal{U}^{A}=\mathcal{H}^{A}=\{0,1\}$. The transition function is given by $f^{A}(a, u)=\left(u \overline{a_{2}}, a_{1}\right)$, and the output function is $h^{A}(a)=a_{2}$. The asynchronous transition graphs $G^{A, 0}$ and $G^{A, 1}$ are:

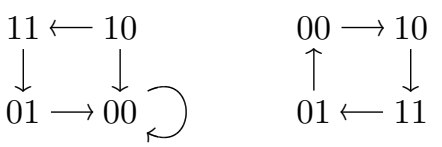

\footnotetext{
${ }^{2}$ We assume only that: (i) at most one variable may be updated at a time, and (ii) every possible transition has a non-zero probability.
} 
The graph $G^{A, 0}$ has four SCCs: $A_{0}^{1}=\{00\}, A_{0}^{2}=\{01\}, A_{0}^{3}=\{10\}$ and $A_{0}^{4}=\{11\} . A_{0}^{1}$ is terminal (it is the attractor of the system under input $u=0$ ) and the other three are transient. The graph $G^{A, 1}$ has only one SCC: $A_{1}^{1}=\{00,01,10,11\}$. It is the attractor of the system under input $u=1$, and it is composed of two non-empty semi-attractors: $A_{1,0}^{1}=\{00,10\}$ and $A_{1,1}^{1}=\{11,01\}$.

\subsection{The interconnection of two Boolean networks}

Let $\Sigma^{A}$ and $\Sigma^{B}$ be two IO ABN, characterized by the objects defined above. The interconnection of $\Sigma^{A}$ and $\Sigma^{B}$ follows the classical definitions from control theory (see for instance [22]), by introducing two feedback functions that transform the outputs of one system into the inputs of the other, as shown in Fig. 1. The resulting network $\Sigma$ is an ABN with no inputs and no outputs. Let $\mu_{A}: \mathcal{H}^{B} \rightarrow \mathcal{U}^{A}$ and $\mu_{B}: \mathcal{H}^{A} \rightarrow \mathcal{U}^{B}$ be two maps. The state space of $\Sigma$ is defined as $\Omega=\Omega^{A} \times \Omega^{B}$ and its transition function $f: \Omega \rightarrow \Omega$ is constructed as: $f(a, b)=\left(f^{A}\left(a, \mu_{A}\left(h^{B}(b)\right), f^{B}\left(b, \mu_{B}\left(h^{A}(a)\right)\right)\right.\right.$. Without loss of generality, it will be assumed that $q_{A}=p_{B}$ and $q_{B}=p_{A}$ and that $\mu_{A}, \mu_{B}$ are in fact identity maps (see Remark 1 below), so that the dynamics of the interconnected system is described by:

$$
\forall x=(a, b) \in \Omega, f(x):=\left(\begin{array}{l}
f^{A}\left(a, h^{B}(b)\right) \\
f^{B}\left(b, h^{A}(a)\right)
\end{array}\right) .
$$

In order to preserve asynchronous dynamics, the transition graph of $\Sigma$, denoted by $G$, is naturally defined as follows. If $x=(a, b)$ and $x^{\prime}=\left(a^{\prime}, b^{\prime}\right)$ are two states in $\Omega^{A} \times \Omega^{B}=\Omega$, then $x^{\prime}$ is a successor of $x$ in the transition graph $G$ (i.e. $\left.x \rightarrow_{G} x^{\prime}\right)$ if and only if:

- either $a=a^{\prime}$ and $b \rightarrow_{G^{B}, h^{A}(a)} b^{\prime}$,

- or $b=b^{\prime}$ and $a \rightarrow_{G^{A, h} h_{(b)}} a^{\prime}$.

Lemma 2 Consider any $a, a^{\prime} \in \Omega^{A}$ and any $b, b^{\prime} \in \Omega^{B}$. Let $\alpha=h^{A}(a)$ and $\beta=h^{B}(b)$. The two assertions hold:

i) If $a \triangleright_{G^{A, \beta}} a^{\prime}$, then $(a, b) \triangleright_{G}\left(a^{\prime}, b\right)$. Consequently, if $a \sim_{G^{A, \beta}} a^{\prime}$, then $(a, b) \sim_{G}\left(a^{\prime}, b\right)$.

ii) If $b \triangleright_{G^{B, \alpha}} b^{\prime}$, then $(a, b) \triangleright_{G}\left(a, b^{\prime}\right)$. Consequently, if $b \sim_{G^{B, \alpha}} b^{\prime}$, then $(a, b) \sim_{G}\left(a, b^{\prime}\right)$.

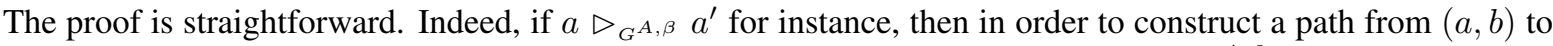
$\left(a^{\prime}, b\right)$ in $G$, it suffices to fix coordinate $b$ (of output $\beta$ ) and let $a$ walk down the graph $G^{A, \beta}$ :

$$
(a, b) \rightarrow_{G}\left(a^{1}, b\right) \rightarrow_{G}\left(a^{2}, b\right) \rightarrow_{G} \ldots \rightarrow_{G}\left(a^{\prime}, b\right) .
$$

Remark 1 The assumption $q_{A}=p_{B}$ and $q_{B}=p_{A}$ has been made for the sake of clarity, but is not a limitation. Indeed, some inputs of $\Sigma^{A}$ or $\Sigma^{B}$ may remain "free" inputs of the interconnected network $\Sigma$ (see Fig. 1). All results would apply similarly, $\Sigma$ being treated as an IO ABN instead of an $A B N$ with no inputs and no outputs.

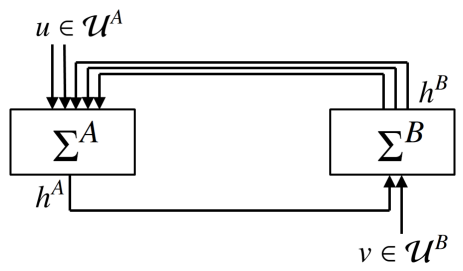

Figure 1: Example of interconnection with $\left(p_{A}, q_{A}\right)=(5,1)$ and $\left(p_{B}, q_{B}\right)=(2,3)$. Three inputs are unplugged.

Example 2 Let $\Sigma^{A}$ be the network described in Example 1 and define $\Sigma^{B}$ as follows: $\Omega^{B}=\{0,1\}^{2}, \mathcal{U}^{B}=$ $\mathcal{H}^{B}=\{0,1\}, f^{B}(b, v)=\left(v \vee b_{2}, b_{1}\right)$ and $h^{B}(b)=b_{2}$. The transition graphs $G^{B, 0}$ and $G^{B, 1}$ are:

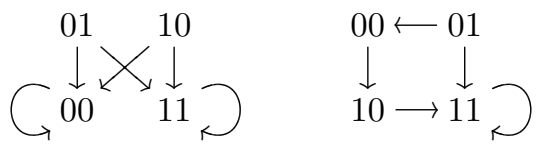


$G^{B, 0}$ has two attractors: $B_{0}^{1}=\{00\}$ and $B_{0}^{4}=\{11\}$, and $G^{B, 1}$ has one attractor: $B_{1}^{4}=\{11\}$. Setting $u=h^{B}(b)=b_{2}$ and $v=h^{A}(a)=a_{2}$, the interconnection $\Sigma$ is a 4-d ABN with no inputs and no outputs, its transition graph $G$ is depicted in Figure 2 .

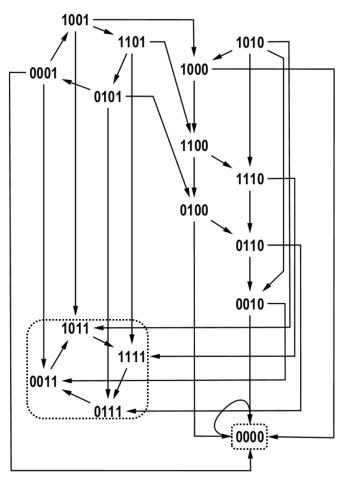

Figure 2: Transition graph of the interconnected network in Example 2. The interconnection has two attractors: the SCC $\{0011,0111,1011,1111\}$ and the steady state $\{0000\}$.

In Sections 4 and 5 we propose and prove a method to predict the attractors of the graph $G$ based on the attractors of $\Sigma^{A}$ and $\Sigma^{B}$. In order to avoid heavy notation, we will restrict ourselves to the interconnection of two Boolean modules. It is important to note that this is not a limitation for the method, and that all following results can be generalized to the interconnection of $k \geq 3$ modules, as long as the computations remain sustainable (see Section 6).

\section{Predicting the attractors of an interconnection}

\subsection{The asymptotic graph}

To motivate the concepts discussed in this section, consider the interconnection of modules $\Sigma^{A}$ and $\Sigma^{B}$ of Example 2. We know that $A_{0,0}^{1}=\{00\}$ is a steady state of $\Sigma^{A}$ under input $u=0$, and $B_{0,0}^{1}=\{00\}$ is a steady state of $\Sigma^{B}$ under input $v=0$. The application of (2) gives:

$$
\begin{aligned}
f\left(A_{0,0}^{1}, B_{0,0}^{1}\right) & =\left(f^{A}\left(A_{0,0}^{1}, 0\right), f^{B}\left(B_{0,0}^{1}, 0\right)\right) \\
& =\left(A_{0,0}^{1}, B_{0,0}^{1}\right),
\end{aligned}
$$

where the last equality follows by definition of the steady states. Therefore, the cross-product $A_{0,0}^{1} \times B_{0,0}^{1}=$ $\{0000\}$ is itself a steady state of the interconnected network $\Sigma$ (it can be verified in Fig. 2). So, one may ask the two following questions: $(i)$ are all cross-products of semi-attractors also attractors of $\Sigma$ ? (ii) Are all attractors of $\Sigma$ of this form? The answer to the first question is, obviously, no. It suffices to consider other cross-products in the previous example, and observe that they are not attractors of $\Sigma$. The answer to the second question is also negative but, in this paper, we show that all the attractors of $\Sigma$ can nevertheless be characterized in terms of these products.

Definition $3 \mathcal{V}^{\text {cr }}$ designates the set of cross-products $A_{u \alpha}^{i} \times B_{v \beta}^{j}, A_{u \alpha}^{i}$ and $B_{v \beta}^{j}$ being respectively non-empty semi-SCCs of $\Sigma^{A}$ under input $u$ and of $\Sigma^{B}$ under input $v$, where $(u, v) \in \mathcal{U}^{A} \times \mathcal{U}^{B},(\alpha, \beta) \in \mathcal{H}^{A} \times \mathcal{H}^{B}$ and where $i$ and $j$ are some integer indices. The set $\mathcal{V}^{\text {as }} \subset \mathcal{V}^{\text {er }}$ is the subset of such products where $A_{u \alpha}^{i}$ and $B_{v \beta}^{j}$ are semi-attractors of $\Sigma^{A}$ and $\Sigma^{B}$.

We now propose the construction of a graph over the elements of $\mathcal{V}^{\text {as }}$, called the asymptotic graph. The idea is to capture the asymptotic behavior of the interconnection by outlining transitions between cross-products of semiattractors. As an example, take two states $a$ and $b$ in semi-attractors $A_{u \alpha}^{i}$ and $B_{v \beta}^{j}$. By adopting an asynchronous 
approach, suppose coordinate $b$ is fixed, while coordinate $a$ evolves in the graph $G^{A, \beta}$. According to Lemma 1, $a$ leads to at least one terminal SCC of $G^{A, \beta}$, say $A_{\beta}^{i^{\prime}}$. Using Lemma 2, the following can be deduced:

$$
A_{u \alpha}^{i} \times B_{v \beta}^{j} \ni(a, b) \triangleright_{G}\left(a^{\prime}, b\right) \in A_{\beta \alpha^{\prime}}^{i^{\prime}} \times B_{v \beta}^{j},
$$

where $\alpha^{\prime}=h^{A}\left(a^{\prime}\right)$. Obviously, a different path is obtained by fixing $a$ and updating $b$, leading to the next definition.

Definition 4 The asymptotic graph of the interconnection $\Sigma$ is the directed graph $\mathcal{G}^{\text {as }}$ whose vertices are elements of $\mathcal{V}^{\text {as }}$ and whose edges are given by:

i) $A_{u \alpha}^{i} \times B_{v \beta}^{j} \rightarrow_{\mathcal{G}^{\text {as }}} A_{\beta \alpha^{\prime}}^{i^{\prime}} \times B_{v \beta}^{j}$ iff there exist $a \in A_{u \alpha}^{i}$ and $a^{\prime} \in A_{\beta \alpha^{\prime}}^{i^{\prime}}$ such that $a \triangleright_{G} A, \beta a^{\prime}$,

ii) $A_{u \alpha}^{i} \times B_{v \beta}^{j} \rightarrow_{\mathcal{G}^{\text {as }}} A_{u \alpha}^{i} \times B_{\alpha \beta^{\prime}}^{j^{\prime}}$ iff there exist $b \in B_{v \beta}^{j}$ and $b^{\prime} \in B_{\alpha \beta^{\prime}}^{j^{\prime}}$ such that $b \triangleright_{G B, \alpha} b^{\prime}$.

Since the number of attractors for graphs $G^{A, u}$ and $G^{B, v}$ are typically much smaller than the total number of states, the graph $\mathcal{G}^{\text {as }}$ is expected to be faster to construct and analyze than the full $G$.

Remark 2 It may happen that two semi-attractors $A_{u, \alpha}^{i}$ and $A_{u^{\prime}, \alpha^{\prime}}^{i^{\prime}}\left(u \neq u^{\prime}\right)$ contain the same states (see for instance $B_{0,1}^{4}$ and $B_{1,1}^{4}$ in Example 3 below). It is yet important to make a clear distinction between them as they correspond to different attractors in different graphs $\left(A_{u}^{i}\right.$ is an attractor of $\Sigma^{A}$ under input $u$ and $A_{u^{\prime}}^{i^{\prime}}$ is an attractor of $\Sigma^{A}$ under input $\left.u^{\prime}\right)$.

Example 3 To illustrate the construction of $\mathcal{G}^{\text {as }}$, consider again the interconnection $\Sigma$ described in Example 2. The semi-attractors of $\Sigma^{A}$ are $A_{0,0}^{1}=\{00\}, A_{1,0}^{1}=\{00,10\}$ and $A_{1,1}^{1}=\{11,01\}$ and the semi-attractors of $\Sigma^{B}$ are $B_{0,0}^{1}=\{00\}, B_{0,1}^{4}=\{11\}$ and $B_{1,1}^{4}=\{11\}$. The set $\mathcal{V}^{\text {as }}$ has $3 \times 3=9$ vertices, which is less than the $2^{4}=16$ states of the full graph $G$. The asymptotic graph $\mathcal{G}^{\text {as }}$ is depicted in Figure 3 . It has two terminal SCCs. The first one is the steady-state $A_{0,0}^{1} \times B_{0,0}^{1}=\{0000\}$ mentioned above. The second one is composed of four vertices. It corresponds to the set of states $\{0111,1111,0011,1011\}$, which is the second attractor of graph $G$ (see Fig. 2).

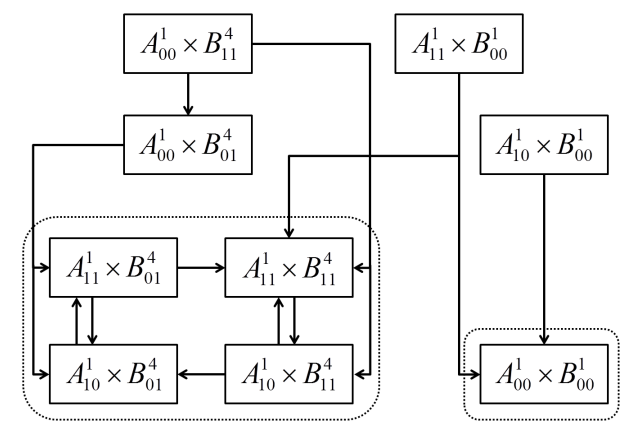

Figure 3: Asymptotic graph of the interconnected network described in Example 2 (for the sake of clarity, selfloops are not represented). The two framed sets are the terminal SCCs.

In this particular example, the asymptotic graph's terminal SCCs capture exactly the attractors of the interconnected network. Next, a theorem is given that establishes and clarifies this relation for any interconnection of two IO ABNs.

Remark 3 It is easy to check that vertices with both $u \neq \beta$ and $v \neq \alpha$ cannot have any incoming edges. Therefore, such vertices cannot belong to terminal SCCs of $\mathcal{G}^{\text {as }}$. To simplify the computations, they can thus be ignored. 


\subsection{Asymptotic dynamics of an interconnection}

For a set $S$, let $\mathfrak{P}(S)$ denote the set of all subsets of $S$. In order to make explicit the correspondence between states and cross-products, we will use two functions $\pi: \mathfrak{P}\left(\mathcal{V}^{\text {cr }}\right) \rightarrow \mathfrak{P}(\Omega)$ and $\psi: \mathfrak{P}(\Omega) \rightarrow \mathfrak{P}\left(\mathcal{V}^{\text {cr }}\right)$ defined as follows.

Definition 5 For $V=A_{u \alpha}^{i} \times B_{v \beta}^{j} \in \mathcal{V}^{\text {cr }}$, let $\pi(V)$ be the set $\pi(V):=\left\{(a, b) \in \Omega: a \in A_{u \alpha}^{i}, b \in B_{v \beta}^{j}\right\} \subset \Omega$. By extension, for $R \subset \mathcal{V}^{\text {cr }}$, let $\pi(R):=\bigcup_{V \in R} \pi(V)$. For $Q \subset \Omega$, let $\psi(Q):=\left\{V \in \mathcal{V}^{\text {cr }}, \pi(V) \subset Q\right\} \subset \mathcal{V}^{\text {cr }}$.

Theorem 1 below establishes the relation between the attractors of an interconnected network $\Sigma$ and the terminal SCCs of its asymptotic graph $\mathcal{G}^{\text {as }}$.

Theorem 1 If $Q$ is an attractor of the interconnected network $\Sigma$, then there exists a terminal SCC $R$ of the asymptotic graph such that $\pi(R) \subset Q$.

A proof of this theorem can be found in [8] but, for completeness, an alternative proof is given in Section 5. In broad terms, Theorem 1 states that every terminal SCC of $G$ generates a terminal SCC in $\mathcal{G}^{\text {as }}$, so the asymptotic graph detects all attractors of $\Sigma$. Moreover, if $Q_{1}$ and $Q_{2}$ are two distinct attractors, then the two corresponding terminal SCCs $R_{1}$ and $R_{2}$ of $\mathcal{G}^{\text {as }}$ are necessarily disjoint. Indeed, the theorem implies that $\pi\left(R_{1}\right) \subset Q_{1}$ and $\pi\left(R_{2}\right) \subset Q_{2}$, and since $Q_{1} \cap Q_{2}=\varnothing$, then automatically $R_{1} \cap R_{2}=\varnothing$. Therefore, all the attractors of $\Sigma$ are unambiguously uncovered by $\mathcal{G}^{\text {as }}$.

Nevertheless, Theorem 1 does not establish a one-to-one correspondence, as $\mathcal{G}^{\text {as }}$ may have more terminal SCCs than $G$. The terminal SCCs that do not correspond to any attractor will be called spurious attractors. An example of an interconnection with a spurious attractor is given in [8] (see also Appendix A). In Section 4.3 the construction of another object, the cross-graph, resolves this issue from a theoretical point of view (see Theorem 2). However, as the construction of the cross-graph may be computationally costly, it would be interesting to have a faster way to decide a priori whether a terminal SCC of $\mathcal{G}^{\text {as }}$ is spurious or not. First, if the asymptotic graph has only one terminal SCC, then it cannot be spurious (it comes from the fact that any graph always has at least one terminal SCC). To go further, Proposition 1 below allows to conclude in some other situations. To state this proposition, introduce the following notation. For a vertex $V=A_{u \alpha}^{i} \times B_{v \beta}^{j}$, let $\pi^{A}(V)$ and $\pi^{B}(V)$ designate respectively the sets $A_{u \alpha}^{i}$ and $B_{v \beta}^{j}$. Then, for $R \subset \mathcal{V}^{\text {as }}$ define the A-output of $R$ as the set $\left\{h^{A}(a) ; a \in \pi^{A}(V), V \in R\right\} \subset \mathcal{H}^{A}$ (similar definition for the $B$-output of $R$ ).

Proposition 1 Let $R$ be a terminal SCC of $\mathcal{G}^{\text {as }}$. If either one of the following conditions is satisfied:

i) $R$ is a singleton;

ii) the A-output of $R$ is a singleton and $S:=\{a, \exists b,(a, b) \in \pi(R)\}$ is an attractor of $G^{A, u}$ for all $u \in \mathcal{U}^{A}$;

iii) the B-output of $R$ is a singleton and $S:=\{b, \exists a,(a, b) \in \pi(R)\}$ is an attractor of $G^{B, v}$ for all $v \in \mathcal{U}^{B}$;

then $\pi(R)$ is equal to an attractor of $\Sigma$.

The proof of this proposition can be found in Appendix B. Note that the terminal SCCs of the asymptotic graph in Example 3 satisfy respectively the first and the last items. So Proposition 1 applies and guarantees that both attractors of Fig. 3 correspond to attractors of the interconnected system (Fig. 2). Furthermore, point i) is particularly relevant for the biological examples of Section 6, since in those examples the attractors of the asymptotic graphs are all singletons.

\subsection{Transient dynamics of an interconnection}

By definition, a transition in the asymptotic graph can only occur when one of subnetworks $\Sigma^{A}$ or $\Sigma^{B}$ has reached an attractor (see Appendix A). It is obviously a strong restriction with respect to the transition graph $G$, where either coordinate $a$ or $b$ can evolve indifferently at each instant. For this reason, some trajectories of $G$ are not reflected in $\mathcal{G}^{\text {as }}$, and spurious attractors may appear. To address this problem, the notion of asymptotic graph can be generalized by taking into account all possible transient dynamics in the space of SCCs, as follows. 
Definition 6 The cross-graph of the interconnection $\Sigma$ is the directed graph $\mathcal{G}^{\text {cr }}$ whose vertices are elements of $\mathcal{V}^{\mathrm{cr}}$ and whose edges are given by:

i) $A_{u \alpha}^{i} \times B_{v \beta}^{j} \rightarrow_{\mathcal{G}^{\text {cr }}} A_{\beta \alpha^{\prime}}^{i^{\prime}} \times B_{v \beta}^{j}$ iff there exist $a \in A_{u \alpha}^{i}$ and $a^{\prime} \in A_{\beta \alpha^{\prime}}^{i^{\prime}}$ such that $a \rightarrow_{G^{A, \beta}} a^{\prime}$,

ii) $A_{u \alpha}^{i} \times B_{v \beta}^{j} \rightarrow_{\mathcal{G}^{\text {cr }}} A_{u \alpha}^{i} \times B_{\alpha \beta^{\prime}}^{j^{\prime}}$ iff there exist $b \in B_{v \beta}^{j}$ and $b^{\prime} \in B_{\alpha \beta^{\prime}}^{j^{\prime}}$ such that $b \rightarrow_{G^{B}, \alpha} b^{\prime}$.

Since semi-attractors are particular semi-SCCs, the set $\mathcal{V}^{\text {as }}$ is included in $\mathcal{V}^{\text {cr. }}$. While the former is expected to be reasonably small, $\mathcal{V}^{\text {cr }}$ can be very large. Even though computable in small examples, the construction of the cross-graph becomes rapidly untractable for large systems. In some cases, it can be larger than the actual transition graph $G$. However, the interest of this graph is rather theoretical than practical, as shown by the following theorem, establishing a one-to-one correspondence between terminal SCCs of $\mathcal{G}^{\mathrm{cr}}$ and of $G$.

Theorem 2 Let $\mathcal{A}(G)$ and $\mathcal{A}\left(\mathcal{G}^{c r}\right)$ denote the set of terminal SCCs of $G$ and $\mathcal{G}^{c r}$, respectively. Then $\pi: \mathcal{A}\left(\mathcal{G}^{c r}\right) \rightarrow$ $\mathcal{A}(G)$ is a bijective function.

In Section 5, we show that Theorem 1 is actually a consequence of Theorem 2, underlying the importance of the cross-graph from a theoretical point of view.

\section{Proof of Theorems 1 and 2}

\subsection{Preliminaries}

Lemma 3 (i) If $Q$ is a SCC of $G$, then $\psi(Q) \neq \emptyset$.

(ii) Any $V \in \mathcal{V}^{\text {cr }}$ of the form $A_{\beta \alpha}^{i} \times B_{\alpha \beta}^{j}$ belongs to $\psi(Q)$, for some SCC $Q$ of $G$.

Proof. (i) $Q$ is a SCC of $G$. Let $x=(a, b) \in Q$ and $\alpha=h^{A}(a), \beta=h^{B}(b)$. By definition, $a$ (resp., $b$ ) belongs to one and only one SCC $A_{\beta}^{i}$ of $G^{A, \beta}$ (resp., $B_{\alpha}^{j}$ of $G^{B, \alpha}$ ). Then, $x \in \pi(V)$ with $V=A_{\beta \alpha}^{i} \times B_{\alpha \beta}^{j}$. Let $x^{\prime}=\left(a^{\prime}, b^{\prime}\right) \in \pi(V) . a, a^{\prime} \in A_{\beta}^{i}$, implying: $a \sim_{G^{A, \beta}} a^{\prime}$. Since $h^{B}(b)=\beta$, Lemma 2 yields $(a, b) \sim_{G}\left(a^{\prime}, b\right)$. Similarly, $b \sim_{G^{B, \alpha}} b^{\prime}$ and $h^{A}\left(a^{\prime}\right)=\alpha$, so $\left(a^{\prime}, b\right) \sim_{G}\left(a^{\prime}, b^{\prime}\right)$. Therefore $x \sim_{G} x^{\prime}$, which proves that $\pi(V)$ is a strongly connected set. In consequence, $\pi(V) \subset Q$ and $V \in \psi(Q)$.

(ii) Consider the vertex $V=A_{\beta \alpha}^{i} \times B_{\alpha \beta}^{j}$, for some $\alpha, \beta, i$ and $j$. If $x=(a, b)$ and $x^{\prime}=\left(a^{\prime}, b^{\prime}\right)$ belong to $\pi(V)$, then Lemma 2 implies $(a, b) \sim_{G}\left(a^{\prime}, b\right) \sim_{G}\left(a^{\prime}, b^{\prime}\right)$. As before, $\pi(V)$ is strongly connected and therefore $V \in \psi(Q)$, where $Q$ is some SCC of $G$.

Lemmas 4 and 5 below establish some relations between paths in $G$ and paths in $\mathcal{G}^{\text {cr }}$.

Lemma 4 Let $V, V^{\prime}$ be two vertices of $\mathcal{G}^{\text {cr }}$ such that $V \triangleright_{\mathcal{G}^{c r}} V^{\prime}$. Then, for any $x^{\prime} \in \pi\left(V^{\prime}\right)$, there exists $x \in \pi(V)$ such that $x \triangleright_{G} x^{\prime}$.

Proof. First assume that $V^{\prime}$ is a successor of $V$. For instance, $V=A_{u \alpha}^{i} \times B_{v \beta}^{j} \rightarrow_{\mathcal{G}^{\text {cr }}} V^{\prime}=A_{\beta \alpha^{\prime}}^{i^{\prime}} \times B_{v \beta}^{j}$ (a similar proof holds for a type ii transition). According to Definition 6, there exist $a_{0} \in A_{u \alpha}^{i}$ and $a_{0}^{\prime} \in A_{\beta \alpha^{\prime}}^{i^{\prime}}$ such that $a_{0} \rightarrow_{G^{A, \beta}} a_{0}^{\prime}$. Let $x^{\prime}=\left(a^{\prime}, b^{\prime}\right)$ be any state in $\pi\left(V^{\prime}\right)$, and let $x:=\left(a_{0}, b^{\prime}\right) \in \Omega$. By definition $x \in \pi(V)$, and $a_{0} \rightarrow_{G^{A, \beta}} a_{0}^{\prime} \sim_{G^{A, \beta}} a^{\prime}$. Since $h_{B}\left(b^{\prime}\right)=\beta$, Lemma 2 yields $x \triangleright_{G} x^{\prime}$.

Now, let $V^{\prime}$ be a descendant of $V$. There exist $k \geq 0$ vertices $V_{1}, \ldots, V_{k} \in \mathcal{V}^{\text {cr }}$ such that $V=V_{1} \rightarrow_{\mathcal{G}^{\text {cr }}} V_{2} \rightarrow_{\mathcal{G}^{\text {cr }}}$ $\ldots \rightarrow{ }_{\mathcal{g}^{\text {cr }}} V_{k}=V^{\prime}$. Let $x^{\prime}$ be any element of $\pi\left(V^{\prime}\right)=\pi\left(V_{k}\right)$. The previous part ensures the existence of $x_{k-1} \in \pi\left(V_{k-1}\right)$ such that $x_{k-1} \triangleright_{G} x^{\prime}$. By applying the same result along the path, one obtains a sequence $\left(x_{l}\right)_{1 \leq l \leq k-1}$ such that $x_{l} \in \pi\left(V_{l}\right)$ and $x_{l} \triangleright_{G} x_{l+1}$. Thus, there is $x=x_{1} \in \pi\left(V_{1}\right)=\pi(V)$ such that $x \triangleright_{G} x^{\prime}$.

Lemma 5 Let $x, x^{\prime} \in \Omega$ such that $x \triangleright_{G} x^{\prime}$. Then, for any $V \in \mathcal{V}^{\text {cr }}$ such that $x \in \pi(V)$, there exists $V^{\prime} \in \mathcal{V}^{\text {cr }}$ such that $x^{\prime} \in \pi\left(V^{\prime}\right)$ and $V \triangleright_{\mathcal{G}^{c r}} V^{\prime}$.

Proof. First, consider $x, x^{\prime}$ such that $x \rightarrow_{G} x^{\prime}$. Let $x=(a, b)$ and $x^{\prime}=\left(a^{\prime}, b^{\prime}\right), \alpha=h_{A}(a)$ and $\beta=h_{B}(b)$. There are two possibilities: either $b=b^{\prime}$ and $a \rightarrow_{G^{A, \beta}} a^{\prime}$, or $a=a^{\prime}$ and $b \rightarrow_{G^{B, \alpha}} b^{\prime}$. Let $V=A_{u \alpha}^{i} \times B_{v \beta}^{j} \in \mathcal{V}^{\text {cr }}$ such that $x \in \pi(V)$. In the first case, we have $V \rightarrow_{\mathcal{G}^{\text {cr }}} V^{\prime}:=\left(A_{\beta \alpha^{\prime}}^{i^{\prime}}, B_{v \beta}^{j}\right)$, where $A_{\beta \alpha^{\prime}}^{i^{\prime}}$ is the semi-SCC of $G^{A, \beta}$ 
containing $a^{\prime}$. In the second case, we have $V \rightarrow_{\mathcal{G}^{\text {cr }}} V^{\prime}:=A_{u \alpha}^{i} \times B_{\alpha \beta^{\prime}}^{j^{\prime}}$, where $B_{\alpha \beta^{\prime}}^{j^{\prime}}$ is the semi-SCC of $G^{B, \alpha}$ containing $b^{\prime}$. Therefore, in both cases we exhibited a vertex $V^{\prime}$ successor of $V$ such that $x^{\prime} \in \pi\left(V^{\prime}\right)$.

Consider now $x, x^{\prime}$ such that $x \triangleright_{G} x^{\prime}$. There are $k$ states $x_{1}, \ldots, x_{k} \in \Omega$ such that $x=x_{1} \rightarrow_{G} x_{2} \rightarrow_{G} \ldots \rightarrow_{G}$ $x_{k}=x^{\prime}$. If $V=V_{1} \in \mathcal{V}^{\text {cr }}$ is such that $x=x_{1} \in \pi\left(V_{1}\right)$, then by applying recursively the previous statement along the path, one finds $\left(V_{l}\right)_{2<l<k}$ such that $x_{l} \in \pi\left(V_{l}\right)$ and $V_{l-1} \rightarrow_{\mathcal{G}^{\text {cr }}} V_{l}$. In particular, the vertex $V^{\prime}=V_{k}$ is such that $x_{k}=x^{\prime} \in \pi\left(V^{\prime}\right)$ and $V \triangleright_{\mathcal{G}^{\text {cr }}} V^{\prime}$.

Finally, we prove some important properties of $\psi(Q)$ and $\pi(R)$, where $Q$ and $R$ are, respectively, terminal SCCs of $G$ and $\mathcal{G}^{\text {cr }}$.

Lemma 6 If $Q \subset \Omega$ is a terminal $S C C$ of $G$, then $\psi(Q)$ is an invariant set of $\mathcal{G}^{c r}$.

Proof. Let $Q$ be a terminal SCC of $G$ and let $V \in \psi(Q)$ (by Lemma 3, $\psi(Q)$ is not empty). Let $V^{\prime}$ be any descendant of $V$ in graph $\mathcal{G}^{\text {cr }}$, and let $y \in \pi\left(V^{\prime}\right)$. Using Lemma 4, there exists $x \in \pi(V)$ such that $x \triangleright_{G} y$. Since $x \in Q$ and $Q$ is terminal, then also $y \in Q$. Therefore, $\pi\left(V^{\prime}\right) \subset Q$, in other words: $V^{\prime} \in \psi(Q)$.

Lemma 7 If $R \subset \mathcal{V}^{\text {cr }}$ is a terminal $S C C$ of $\mathcal{G}^{c r}$. Then $\pi(R)$ is an invariant set of $G$.

Proof. Let $R$ be a terminal SCC of $\mathcal{G}^{\text {cr }}$ and let $x \in \pi(R)$. By definition, there exists $V \in R$ such that $x \in \pi(V)$. Now consider any descendant $x^{\prime}$ of $x$. Lemma 5 implies there is $V^{\prime} \in \mathcal{V}^{\text {cr }}$ such that $V \triangleright_{\mathcal{G}^{\text {cr }}} V^{\prime}$ and $x^{\prime} \in \pi\left(V^{\prime}\right)$. Since $R$ is terminal, $V^{\prime} \in R$ and $x^{\prime} \in \pi(R)$.

\subsection{Proof of Theorem 2}

Recall that $\mathcal{A}\left(\mathcal{G}^{\text {cr }}\right) \subset \mathfrak{P}\left(\mathcal{V}^{\text {cr }}\right)$ and $\mathcal{A}(G) \subset \mathfrak{P}(\Omega)$ denote respectively the sets of attractors of $\mathcal{G}^{\text {cr }}$ and $G$. Theorem 2 ensues from the three following results: the image of $\mathcal{A}\left(\mathcal{G}^{\text {cr }}\right)$ by $\pi$ is included in $\mathcal{A}(G)$ (Lemma 8), the map $\pi: \mathcal{A}\left(\mathcal{G}^{\text {cr }}\right) \rightarrow \mathcal{A}(G)$ is surjective (Lemma 9) and injective (Lemma 10).

Lemma 8 If $R$ is a terminal SCC of $\mathcal{G}^{c r}$, then $\pi(R)$ is a terminal SCC of $G$.

Proof. Let $R \in \mathcal{A}\left(\mathcal{G}^{\mathrm{cr}}\right)$. According to Lemma 7, the set $\pi(R)$ is an invariant of $G$. As a consequence, there is $Q \in \mathcal{A}(G)$ such that $Q \subset \pi(R)$ (Lemma 1). To show that this inclusion is in fact an equality, consider $x=(a, b) \in Q$, with $\alpha=h^{A}(a), \beta=h^{B}(b)$. Since $Q \subset \pi(R)$, there exists $V=A_{u \alpha}^{i} \times B_{v \beta}^{j} \in R$ such that $\pi(V) \ni x$. Note that $u=\beta$ or $v=\alpha$ (Remark 3 ), which leads to consider three cases.

Case 1. If both $u=\beta$ and $v=\alpha$, then $V$ belongs to some $\psi\left(Q^{\prime}\right)$, where $Q^{\prime}$ is a SCC of $G$ (Lemma 3). Therefore, we have $x \in Q \cap Q^{\prime}$, which implies $Q^{\prime}=Q$ and $V \in \psi(Q)$.

Case 2. If $u=\beta$ and $v \neq \alpha$, then $b \in B_{v \beta}^{j}$ has descendants in a semi-attractor of $G^{B, \alpha}$, say: $B_{\alpha \beta^{\prime}}^{j^{\prime}}$. We have $V \triangleright_{\mathcal{G}^{\text {cr }}} V^{\prime}=A_{\beta \alpha}^{i} \times B_{\alpha \beta^{\prime}}^{j^{\prime}}$, so $V^{\prime} \in R$ (since $R$ is terminal). For any state $x^{\prime}=\left(a^{\prime}, b^{\prime}\right) \in \pi\left(V^{\prime}\right)$, the following two steps show that $x^{\prime}$ is actually a descendant of $x$ in $G$. First, $a, a^{\prime} \in A_{\beta \alpha}^{i}$ and $h^{B}(b)=\beta$ so $(a, b) \sim_{G}\left(a^{\prime}, b\right)$. Second, $b \triangleright_{G B, \alpha} b^{\prime} \in B_{\alpha \beta^{\prime}}^{j^{\prime}}$ and $h^{A}\left(a^{\prime}\right)=\alpha$, so $\left(a^{\prime}, b\right) \triangleright_{G}\left(a^{\prime}, b^{\prime}\right)$. Therefore, $x^{\prime} \in Q$ (since $Q$ is terminal) and $V^{\prime} \in \psi(Q)$.

Case 3. If $u \neq \beta$ and $v=\alpha$, then by a similar reasoning, there is $V^{\prime}=A_{\beta \alpha^{\prime}}^{i^{\prime}} \times B_{v \beta}^{j}$ in $R$ that belongs to $\psi(Q)$. In all cases, we exhibited a vertex of $R$ that lies in $\psi(Q)$. Since $Q$ is terminal, $\psi(Q)$ is an invariant of $\mathcal{G}^{\text {cr }}$ (Lemma 6), implying $R \subset \psi(Q)$ and so $\pi(R) \subset Q$.

Lemma 9 [Surjectivity] For every terminal SCC $Q$ of $G$, there exists a terminal SCC $R$ of $\mathcal{G}^{\text {cr }}$ such that $\pi(R)=$ $Q$.

Proof. Let $Q \in \mathcal{A}(G)$. According to Lemma $6, \psi(Q)$ is an invariant of $\mathcal{G}^{\mathrm{cr}}$, so it contains at least one terminal SCC $R$ of $\mathcal{G}^{\text {cr }}$ (Lemma 1). We have $R \subset \psi(Q)$, so $\pi(R) \subset Q$. Moreover, Lemma 8 implies that $\pi(R) \in \mathcal{A}(G)$, therefore, $\pi(R) \equiv Q$.

Lemma 10 [Injectivity] If $R_{1}, R_{2}$ are two distinct terminal SCCs of $\mathcal{G}^{c r}$, then $\pi\left(R_{1}\right) \neq \pi\left(R_{2}\right)$. 
Proof. Let $R_{1}, R_{2} \in \mathcal{A}\left(\mathcal{G}^{\text {cr }}\right)$ such that $\pi\left(R_{1}\right)=\pi\left(R_{2}\right)=Q$ and let $x=(a, b) \in Q$, with $\alpha=h^{A}(a)$, $\beta=h^{B}(b)$. There are $V_{1}=A_{u_{1} \alpha}^{i_{1}} \times B_{v_{1} \beta}^{j_{1}} \in R_{1}$ and $V_{2}=A_{u_{2} \alpha}^{i_{2}} \times B_{v_{2} \beta}^{j_{2}} \in R_{2}$ such that $x \in \pi\left(V_{1}\right) \cap \pi\left(V_{2}\right)$. By Lemma 1, there exists a terminal SCC of $G^{A, \beta}$, say $A_{\beta}^{i^{\prime}}$, and $a^{\prime} \in A_{\beta}^{i^{\prime}}$ such that $a \triangleright_{G^{A, \beta}} a^{\prime}$, implying $x \triangleright_{G}\left(a^{\prime}, b\right)$. Setting $\alpha^{\prime}=h_{A}\left(a^{\prime}\right)$ and choosing to update only coordinate $a$, one constructs the two paths $V_{1} \triangleright_{\mathcal{G}^{\text {cr }}} A_{\beta \alpha^{\prime}}^{i^{\prime}} \times B_{v_{1} \beta}^{j_{1}}$ and $V_{2} \triangleright_{\mathcal{G}^{\text {cr }}} A_{\beta \alpha^{\prime}}^{i^{\prime}} \times B_{v_{2} \beta}^{j_{2}}$ in $\mathcal{G}^{\text {cr }}$. Consider now $b$ in graph $G^{B, \alpha^{\prime}}$ : there exists $b^{\prime} \in B_{\alpha^{\prime}}^{j^{\prime}}$ (a terminal SCC) such that $b \triangleright_{G B, \alpha^{\prime}} b^{\prime}$, implying $\left(a^{\prime}, b\right) \triangleright_{G}\left(a^{\prime}, b^{\prime}\right)$. Setting $\beta^{\prime}=h_{B}\left(b^{\prime}\right)$ and choosing to update coordinate $b$, one constructs the two paths: $A_{\beta \alpha^{\prime}}^{i^{\prime}} \times B_{v_{1} \beta}^{j_{1}} \triangleright_{\mathcal{G}^{\text {cr }}} A_{\beta \alpha^{\prime}}^{i^{\prime}} \times B_{\alpha^{\prime} \beta^{\prime}}^{j^{\prime}}$ and $A_{\beta \alpha^{\prime}}^{i^{\prime}} \times B_{v_{2} \beta}^{j_{2}} \triangleright_{\mathcal{G}^{\text {cr }}} A_{\beta \alpha^{\prime}}^{i^{\prime}} \times B_{\alpha^{\prime} \beta^{\prime}}^{j^{\prime}}$. Therefore, $A_{\beta \alpha^{\prime}}^{i^{\prime}} \times B_{\alpha^{\prime} \beta^{\prime}}^{j^{\prime}}$ is a descendant of $V_{1}$ and $V_{2}$ so it belongs to $R_{1}$ and to $R_{2}$ (because $R_{1}$ and $R_{2}$ are terminal). Since distinct SCCs are necessarily disjoint, we have $R_{1}=R_{2}$.

\subsection{Proof of Theorem 1}

The following lemma establishes some correspondence between paths of $\mathcal{G}^{\text {as }}$ and $\mathcal{G}^{\text {cr }}$.

Lemma 11 Let $V, V^{\prime}$ designate two vertices of $\mathcal{V}^{\text {as }}$.

i) If $V \triangleright_{\mathcal{G}^{a s}} V^{\prime}$, then $V \triangleright_{\mathcal{G}^{c r}} V^{\prime}$.

ii) If $V \rightarrow \operatorname{gg}^{\text {cr }} V^{\prime}$, then $V \rightarrow \operatorname{g}^{a s} V^{\prime}$.

Remark that the existence of a path $V \triangleright_{\mathcal{G}^{\text {cr }}} V^{\prime}$ does not necessarily imply $V \triangleright_{\mathcal{G}^{\text {as }}} V^{\prime}$ (see Appendix A).

Proof. i) First suppose that $V \rightarrow_{\mathcal{G}^{\text {as }}} V^{\prime}$, i.e. $V^{\prime}$ is a successor of $V$ in $\mathcal{G}^{\text {as }}$. For instance $V=A_{u \alpha}^{i} \times B_{v \beta}^{j}$ and $V^{\prime}=A_{\beta \alpha^{\prime}}^{i^{\prime}} \times B_{v \beta}^{j}$ (a similar proof will hold for a type ii edge). Then according to Definition 4, there are $a \in A_{u \alpha}^{i}$ and $a^{\prime} \in A_{\beta \alpha^{\prime}}^{i^{\prime}}$ such that $a \rightarrow_{G^{A, \beta}} a_{1} \rightarrow_{G^{A, \beta}} \ldots \rightarrow_{G^{A, \beta}} a_{k} \rightarrow_{G^{A, \beta}} a^{\prime}$. By following this path in $G^{A, \beta}$, one deduces the following path in $\mathcal{G}^{\text {cr: }}$

$$
\begin{array}{rllll}
A_{u \alpha}^{i} \times B_{v \beta}^{j} & \rightarrow_{\mathcal{G}^{\text {cr }}} & A_{\beta \alpha_{1}}^{i_{1}} \times B_{v \beta}^{j} & \rightarrow_{\mathcal{G}^{\text {cr }}} & \ldots \\
\ldots & \rightarrow_{\mathcal{G}^{\text {cr }}} & A_{\beta \alpha_{k}}^{i_{k}} \times B_{v \beta}^{j} & \rightarrow_{\mathcal{G}^{\text {cr }}} & A_{\beta \alpha^{\prime}}^{i^{\prime}} \times B_{v \beta}^{j},
\end{array}
$$

where, for each $l, \alpha_{l}=h_{A}\left(a_{l}\right)$ and $A_{\beta}^{i_{l}}$ is the SCC of $G^{A, \beta}$ that contains $a_{l}$. As a result, $V \triangleright_{\mathcal{G}^{\text {cr }}} V^{\prime}$.

Suppose now that $V^{\prime}$ is a descendant of $V$ in $\mathcal{G}^{\text {as }}: V \rightarrow_{\mathcal{G}^{\text {as }}} V_{1} \rightarrow_{\mathcal{G}^{\text {as }}} \ldots \rightarrow_{\mathcal{G}^{a s}} V_{k} \rightarrow_{\mathcal{G}^{a s}} V^{\prime}$. Then, applying the result above to each edge leads to $V \triangleright_{\mathcal{G}^{\text {cr }}} V^{\prime}$.

ii) Let $V, V^{\prime} \in \mathcal{V}^{\text {as }}$ such that $V \rightarrow_{\mathcal{G}^{\text {cr }}} V^{\prime}$, for instance $V=A_{u \alpha}^{i} \times B_{v \beta}^{j}$ and $V^{\prime}=A_{\beta \alpha^{\prime}}^{i^{\prime}} \times B_{v \beta}^{j}$ (a similar proof will hold for a type ii edge). Then by Definition 6, there are $a \in A_{u \alpha}^{i}$ and $a^{\prime} \in A_{\beta \alpha^{\prime}}^{i^{\prime}}$ such that $a \rightarrow_{G^{A, \beta}} a^{\prime}$ which implies, according to Definition $4, V \rightarrow_{\mathcal{G}^{\text {as }}} V^{\prime}$.

Lemma 12 If $R$ is a terminal SCC of $\mathcal{G}^{c r}$, then $R \cap \mathcal{V}^{\text {as }}$ is not empty. Furthermore $R \cap \mathcal{V}^{a s}$ is an invariant of $\mathcal{G}^{a s}$.

Proof. Let $R$ be a terminal SCC of $\mathcal{G}^{\mathrm{cr}}$, and let $V=A_{u \alpha}^{i} \times B_{v \beta}^{j}$ denote an element of $R$. Under input $\beta$, any $a \in A_{u \alpha}^{i}$, eventually reaches some semi-attractor $A_{\beta \alpha^{\prime}}^{i^{\prime}}$. By definition of the cross-graph, $V \triangleright_{\mathcal{G}^{\text {cr }}} A_{\beta \alpha^{\prime}}^{i^{\prime}} \times B_{v \beta}^{j}$. Consider now any $b \in B_{v \beta}^{j}$, which will eventually reach some semi-attractor $B_{\alpha^{\prime} \beta^{\prime}}^{j^{\prime}}$. Therefore, $A_{\beta \alpha^{\prime}}^{i^{\prime}} \times B_{v \beta}^{j} \triangleright_{\mathcal{G}^{\text {cr }}}$ $A_{\beta \alpha^{\prime}}^{i^{\prime}} \times B_{\alpha^{\prime} \beta^{\prime}}^{j^{\prime}}=V^{\prime}$. By construction, this vertex $V^{\prime}$ belongs to $\mathcal{V}^{\text {as }}$ as $A_{\beta \alpha^{\prime}}^{i^{\prime}}$ and $B_{\alpha^{\prime} \beta^{\prime}}^{j^{\prime}}$ are semi-attractors of graphs $G^{A, \beta}$ and $G^{B, \alpha^{\prime}}$ respectively. In conclusion, we exhibited a descendant $V^{\prime}$ of $V$ (in $\mathcal{G}^{\text {cr }}$ ) that belongs to $\mathcal{V}^{\text {as }}$. Since $R$ is terminal, $V^{\prime} \in R$ which proves that $R \cap \mathcal{V}^{\text {as }}$ is not empty.

In order to prove the second part, consider a vertex $V \in R \cap \mathcal{V}^{\text {as }}$, and let $V^{\prime}$ designate any descendant of $V$ in $\mathcal{G}^{\text {as }}$, i.e.: $V^{\prime} \in \mathcal{V}^{\text {as }}$ and $V \triangleright_{\mathcal{G}^{\text {as }}} V^{\prime}$. Lemma 11 implies $V \triangleright_{\mathcal{G}^{\text {cr }}} V^{\prime}$, which implies that $V^{\prime} \in R$ (since $R$ is a terminal SCC of $\mathcal{G}^{\text {cr }}$ ). Therefore, $V^{\prime}$ lies in $R \cap \mathcal{V}^{\text {as }}$.

Proof. (Theorem 1) Let $Q$ be an attractor of $G$. According to Theorem 2, there exists a unique $R$, terminal SCC of $\mathcal{G}^{\text {cr }}$ such that $\pi(R)=Q$. By Lemma 12 , the set $R \cap \mathcal{V}^{\text {as }}$ is nonempty and an invariant of $\mathcal{G}^{\text {as }}$. Therefore, there exists a terminal SCC $R^{\prime}$ of $\mathcal{G}^{\text {as }}$ such that $R^{\prime} \subset R$. We further have $\pi\left(R^{\prime}\right) \subset \pi(R)=Q$. 


\section{Example of application: multicellular systems}

The main interest of Theorem 1 is to rapidly obtain the asymptotic dynamics of a network through the analysis of separate modules. Given the generally high degree of modularity observed in biology [15], it thus provides a suitable method to identify the asymptotic behaviors of possibly large biological systems. The analysis of multicellular systems, for example, constitutes a relevant illustration of this method. To model such systems, a basic way consists in interconnecting several copies of the same network, thus mimicking a group of cells communicating with each other (for instance through the diffusion of a key signalling molecule). A classical example is the segment polarity network in the fruit fly (Drosophila melanogaster). Segment polarity genes are involved in embryogenesis, and in particular in the formation of patterns within the embryo. Several discrete models have been proposed during the past decade $[2,6,20]$, generally considering unidimensional, four to six cell wide embryo segments, so that each cell communicates with its left and right neighbors.

A first Boolean model has been developed in [2]; it consists of a four cell segment with periodic boundary conditions. There are essentially five genes which follow a similar network of interactions in each cell, but also receive stimulus from two proteins in nearest cells. The definition of the network can be found in Appendix C, the reader is also referred to [2], references therein, and $[6,7]$ for further details on the model. By considering the whole segment as a unique network, one would have to build and analyze a transition graph of $2^{7 \times 4} \approx$ $2.7 \times 10^{8}$ states, whereas the asymptotic graph of the interconnection has only 7744 vertices (barely 3768 by implementing the reduction method suggested in Remark 3). A rapid analysis shows that its terminal SCCs are all singletons. Therefore, Theorem 1 and Proposition 1 imply they are all attractors of the interconnected system. These attractors are not reproduced here, but they correspond to the steady states found in [2] and represent different phenotypes, including the wild type, a ptc knock-out mutant, an en knock-out mutant, and some ectopic pattern variations. With respect to [2], the use of the asymptotic graph allows to conclude that those attractors are actually the only possible asymptotic behaviors of the four cell segment (which could not be deduced by using only simulations).

Remark 4 As evoked earlier, the definition of the asymptotic graph can be easily generalized for more than two modules. In this case for instance, its vertices will be of the form $A_{u, \alpha}^{i} \times B_{v, \beta}^{j} \times C_{w, \gamma}^{k} \times D_{z, \delta}^{l}$ (with obvious notations), and each vertex will have up to four different kinds of successors, obtained by updating each module separately. For instance, if $\mu: \mathcal{H}^{B} \times \mathcal{H}^{C} \times \mathcal{H}^{D} \rightarrow \mathcal{U}^{A}$ designates the interconnection map of the first module, then the following transition:

$$
A_{u, \alpha}^{i} \times B_{v, \beta}^{j} \times C_{w, \gamma}^{k} \times D_{z, \delta}^{l} \longrightarrow A_{u^{\prime}, \alpha^{\prime}}^{i^{\prime}} \times B_{v, \beta}^{j} \times C_{w, \gamma}^{k} \times D_{z, \delta}^{l}
$$

will exist iff there exist $a \in A_{u, \alpha}^{i}$ and $a^{\prime} \in A_{u^{\prime}, \alpha^{\prime}}^{i^{\prime}}$ such that $a \triangleright_{G^{A, u^{\prime}}} a^{\prime}$ (where $u^{\prime}=\mu(\beta, \gamma, \delta)$ and $\alpha^{\prime}=h^{A}\left(a^{\prime}\right)$ ).

Another discrete model of the same system has been proposed in [20], based on a slightly different interaction network. While technically not Boolean (there are some ternary variables), it was still possible to translate this model into a 13-dimensional IO ABN with 4 inputs using the technique presented in [9] (not shown). As before, we were able to compute the asymptotic graph of a four cell segment. It contains 47089 vertices (23813 after reduction), which is far less than the whole transition graph (which contains $2^{13 \times 4} \approx 4.5 \times 10^{15}$ states). Once again, this graph contains only single-vertex terminal SCCs, ensuring we have unambiguously recovered all asymptotic patterns of the segment.

\section{Towards a model reduction technique}

The previous examples showed that the use of the asymptotic graph can drastically reduce the computational cost of the search for attractors. Given that the size of a transition graph grows exponentially with the network's dimension, this reduction is crucial and can sometimes be determinant to computationally achieve this search in reasonable time. Thus, the asymptotic graph can also provide a model reduction technique by decomposing a large network into two (or more), computationally tractable modules. Contrary to other techniques (see e.g. [1]), the asymptotic graph method is not restricted to the search of singleton attractors and is adapted to asynchronous networks which, as mentioned earlier, generate nondeterministic dynamics that are more complex than in the synchronous case. It provides an alternative to the method in [18], by focusing on the modularity of cellular networks. 
Table 1: Comparison of three classical partitioning algorithms.

\begin{tabular}{|l|ccc|ccc|c|c|}
\cline { 2 - 8 } \multicolumn{1}{c|}{} & $n_{1}$ & $p_{1}$ & $q_{1}$ & $n_{2}$ & $p_{2}$ & $q_{2}$ & $m$ & $\Gamma_{1}$ \\
\hline hierarchical & 6 & 2 & 3 & 16 & 3 & 2 & 5 & 524544 \\
MCL & 6 & 2 & 3 & 16 & 3 & 2 & 5 & 524544 \\
spectral & 9 & 3 & 3 & 13 & 3 & 3 & 6 & 69632 \\
\hline
\end{tabular}

In general, the efficiency of this reduction technique will depend on a balance between the number and size of the modules and the number of inputs. To formalize this idea, consider an ABN $\Sigma$ with $n$ variables $\mathcal{X}=\left\{x_{1}, \ldots, x_{n}\right\}$ and a $k$-partition $\mathcal{P}=\left(\mathcal{X}_{1}, \ldots, \mathcal{X}_{k}\right)$ of $\mathcal{X}$ with $n_{i}:=\left|\mathcal{X}_{i}\right|, 1 \leq i \leq k\left(n_{1}+\ldots+n_{k}=n\right)$. This partition generates $k$ IO modules $A_{i}$ of dimensions $n_{i}$. $\Sigma$ can be seen as the interconnection of the $A_{i}$ along a certain interconnection scheme, given by the logical rules of $\Sigma$. Let $p_{i}$ and $q_{i}$ denote respectively the number of inputs and outputs of module $A_{i}$. Hereafter, we make the assumption that the cost of the analysis of a graph (i.e. SCC decomposition, identification of terminal SCCs) amounts to its size. With this simplifying assumption, the direct search for the attractors of $\Sigma$ would cost $2^{n}$. With decomposition $\mathcal{P}$, the search for attractors involves two successive steps: the analyses of the modules (each $A_{i}$ needs the computation of $2^{p_{i}}$ transition graphs of size $\left.2^{n_{i}}\right)$, and the analysis of the asymptotic graph, leading to a total cost of

$$
\Gamma(\mathcal{P})=\underbrace{\sum_{i=1}^{k} 2^{p_{i}+n_{i}}}_{\Gamma_{1}}+\underbrace{\prod_{i=1}^{k}\left(\sum_{u \in\{0,1\}^{p_{i}}} \sum_{\alpha \in\{0,1\}^{q_{i}}} N_{u, \alpha}^{i}\right)}_{\Gamma_{2}},
$$

where $N_{u, \alpha}^{i}$ is the number of semi-attractors of module $A_{i}$ under input $u$ with output $\alpha$. The first term of this sum will be small if the partition is balanced and if the total number of inputs $p:=\sum_{i=1}^{k} p_{i}$ is small. The second term is harder to assess, because the $N_{u, \alpha}^{i}$ depend on the dynamics of each module. However, by considering the best case scenario (each transition graph has only one attractor, with a fixed output), we obtain the following lower bound:

$$
\prod_{i=1}^{k}\left(\sum_{u} \sum_{\alpha} N_{u, \alpha}^{i}\right) \geq \prod_{i=1}^{k} 2^{p_{i}}=2^{p} .
$$

Even though this bound may be far from the actual size, note that it directly depends on the number of inputs $p$, confirming this parameter is critical for the decomposition to be computationally efficient.

The problem of finding a partition $\mathcal{P}$ that minimizes the objective function $\Gamma$ is a typical graph partitioning problem. This type of problems are known to be NP-hard in general, nevertheless a large variety of efficient algorithms exist in the literature, generally aiming at minimizing the edges between modules. Three notable examples are spectral techniques [25], hierarchical clustering [16] and Markov cluster algorithm (MCL) [24]. In order to test those algorithms for our problem, we used a cell fate decision model originally proposed in [5] (see also [8]). Each algorithm was applied to the 22-dimensional network to decompose it into two modules $A_{1}$ and $A_{2}$. We then computed the first term $\Gamma_{1}$ of formula (3). The results are summarized in Table 1. In this table, $m$ denotes the size of the cut, i.e. the number of edges between modules. Observe that while MCL and hierarchical clustering seem more efficient in minimizing this parameter, spectral clustering tend to give a more balanced decomposition, leading to a much lower cost $\Gamma_{1}$. We used the latter to construct the asymptotic graph: its size is $\Gamma_{2}=96$, amounting to a total cost $\Gamma=69728$, which is far less than $2^{22} \approx 4.2 \times 10^{6}$. Further analysis of this graph reveals that it contains no spurious attractors, implying we recovered exactly the attractors of the original network.

If classical algorithms seem to give an efficient decomposition, note that it is not guaranteed to be the best one. Given the relative small size of the example above, we managed to enumerate all perfectly balanced partitions (i.e. with $n_{1}=n_{2}=11$ ) and found even better decompositions (an example of which can be found in [8]). One reason for this is that general partitioning algorithms are designed to minimize the size $m$ of the cut, whereas the critical parameter to minimize here is rather the total number of inputs $p$. An adaptation of these algorithms to the criterion $\Gamma$ is currently under progress. Finally, note that even though two decompositions will lead to two different asymptotic graphs, Theorem 1 ensures that both graphs will capture the attractors of the interconnection. The difference is that the spurious attractors may not be the same, as observed in some examples (not shown here), which further suggests a method to extend Proposition 1. 


\section{Conclusion}

Two new objects, the asymptotic and cross- graphs, have been introduced to characterize the dynamics of an interconnection of two (or more) IO ABNs. These graphs are based exlusively on information available on the individual modules (namely, their strongly connected components) and on the interconnection function, and recover all the asymptotic behaviors of the full interconnected system. Furthermore, it was shown on several examples that the asymptotic graph (which uses only the terminal SCCs of each module) can greatly reduce the computational cost generally associated with the search for attractors.

This graph gives rise to a new technique to analyze a composition of individual modules (such as multicellular biological networks). It also provides a way to study a large network provided it can be decomposed into loosely interconnected modules. Both approaches have been illustrated with biological examples, showing the analytic and computational strength of the asymptotic graph: in each case all attractors have been recovered, and the absence of periodic attractors has been proved, a fact that was not possible to establish with simulation-based analyses.

Our results suggest several research directions to explore, the main one being the adaptation of well known graph partitioning techniques to find cost-efficient decompositions of an ABN. This point is critical to balance the simplifying power of the method with the multiplication of the number of inputs, in order to increase the effectiveness of such a model reduction technique.

\section{Acknowledgments}

MC was supported in part by the french National Research Agency (project GeMCo, ANR 2010 BLAN0201-01).

\section{References}

[1] T. Akutsu, A.A. Melkman, T. Tamura, and M. Yamamoto. Determining a singleton attractor of a Boolean network with nested canalyzing functions. J. Comput. Biol., 18(10):1275-1290, 2011.

[2] R. Albert and G. Othmer. The topology of the regulatory interactions predicts the expression pattern of the Drosophila segment polarity genes. J. Theor. Biol., 223:1-18, 2003.

[3] D. Angeli and E.D. Sontag. Monotone control systems. IEEE Trans. Automat. Contr., 48(10):1684 - 1698, 2003.

[4] S. Bornholdt. Boolean network models of cellular regulation: prospects and limitations. J. R. Soc. Interface, 5:S85-S94, 2008.

[5] L. Calzone, L. Tournier, S. Fourquet, D. Thieffry, B. Zhivotovsky, E. Barillot, and A. Zinovyev. Mathematical modelling of cell-fate decision in response to death receptor engagement. PLoS Comput. Biol., 6(3):e1000702, 2010.

[6] M. Chaves and R. Albert. Studying the effect of cell division on expression patterns of the segment polarity genes. J. R. Soc. Interface, 5:S71-S84, 2008.

[7] M. Chaves, R. Albert, and E.D. Sontag. Robustness and fragility of boolean models for genetic regulatory networks. J. Theor. Biol., 235:431-449, 2005.

[8] M. Chaves and L. Tournier. Predicting the asymptotic dynamics of large biological networks by interconnections of Boolean modules. In Proc. IEEE Conf. Decision and Control, Orlando, USA, 2011.

[9] M. Chaves, L. Tournier, and J.L. Gouzé. Comparing Boolean and piecewise affine differential models for genetic networks. Acta biotheoretica, 58(2):217-232, 2010.

[10] D. Cheng and H. Qi. Controllability and observability of Boolean control networks. Automatica, 45:16591667, 2009. 
[11] T.H. Cormen, C.E. Leiserson, R.L. Rivest, and C. Stein. Introduction to algorithms. MIT Press and McGraw-Hill, 2001.

[12] A. Fauré, A. Naldi, C. Chaouiya, and D. Thieffry. Dynamical analysis of a generic Boolean model for the control of the mammalian cell cycle. Bioinformatics, 22(14):e124-e131, 2006.

[13] G. Goessler. Component-based modeling and reachability analysis of genetic networks. IEEE/ACM Trans. Comput. Biol. Bioinform., 8(3):672-682, 2011.

[14] M. Gondran and M. Minoux. Graphs and algorithms. Wiley \& sons, 1984.

[15] L.H. Hartwell, J.J. Hopfield, S. Leibler, and A.W. Murray. From molecular to modular cell biology. Nature, 402(6761):47, 1999.

[16] S.C. Johnson. Hierarchical clustering schemes. Psychometrika, 32(3):241-254, 1967.

[17] F. Li, T. Long, Y. Lu, Q. Ouyang, and C. Tang. The yeast cell-cycle network is robustly designed. PNAS, 101(14):4781-4786, 2004.

[18] A. Naldi, E. Rémy, D. Thieffry, and C. Chaouiya. Dynamically consistent reduction of logical regulatory graphs. Theor. Comput. Sci., 412(21):2207-18, 2011.

[19] J.A. Papin, T. Hunter, B.O. Palsson, and S. Subramaniam. Reconstruction of cellular signalling networks and analysis of their properties. Nature Rev. Mol. Cell. Biol., 6(2):99-111, 2005.

[20] L. Sánchez, C. Chaouiya, and D. Thieffry. Segmenting the fly embryo: logical analysis of the role of the segment polarity cross-regulatory module. Int. J. Dev. Biol., 52(8):1059, 2008.

[21] R. Schlatter, K. Schmich, I. Avalos Vizcarra, P. Scheurich, T. Sauter, et al. ON/OFF and beyond - a Boolean model of apoptosis. PLoS Comput. Biol., 5(12):e1000595, 2009.

[22] E.D. Sontag. Mathematical Control Theory. Deterministic Finite-Dimensional Systems. Springer-Verlag, New York, 1998.

[23] L. Tournier and M. Chaves. Uncovering operational interactions in genetic networks using asynchronous Boolean dynamics. J. Theor. Biol., 260(2):196-209, 2009.

[24] S. Van Dongen. Graph clustering via a discrete uncoupling process. SIAM J. Matrix Anal. \& Appl., 30(1):121-141, 2008.

[25] U. von Luxburg. A tutorial on spectral clustering. Stat. Comput., 17:395-416, 2007.

[26] R. Zhang, M.V. Shah, J. Yang, S.B. Nyland, X. Liu, J.K. Yun, R. Albert, and T.P. Loughran Jr. Network model of survival signaling in LGL leukemia. PNAS, 105:16308-16313, 2008.

\section{A An example of a spurious attractor}

Let $\Sigma^{A}$ and $\Sigma^{B}$ be two 3-dimensional SISO ABNs, characterized by the transition functions:

$$
\left\{\begin{array}{l}
f_{1}^{A}=\overline{\overline{a_{1}}} a_{2} \overline{\overline{a_{3}}} \vee u \overline{a_{2}}\left(a_{1} \vee a_{3}\right) \vee \bar{u} a_{1} a_{2} a_{3}, \\
f_{2}^{A}=\overline{a_{1}} \overline{a_{3}} \vee u a_{2} \vee a_{1} a_{2}, \\
f_{3}^{A}=\left(\overline{a_{1}} \overline{a_{2}} \vee a_{1} a_{2}\right)\left(\bar{u} a_{3} \vee u \overline{a_{3}}\right) \vee \overline{a_{1}} a_{2}\left(\bar{u} \overline{a_{3}} \vee u a_{3}\right) \vee \bar{u} a_{1} \overline{a_{2}} \overline{a_{3}}, \\
f_{1}^{B}=\overline{b_{1}} b_{3}\left(\overline{b_{2}} \vee \bar{v}\right) \vee b_{2} \overline{b_{3}} \vee v b_{1} \overline{b_{3}}, \\
f_{2}^{B}=\bar{v} \overline{b_{2}} \overline{b_{3}} \vee \overline{b_{1}} \overline{b_{2}} b_{3} \vee v \overline{b_{1}} b_{2} \overline{b_{3}} \vee \bar{v} b_{3} \vee b_{1} b_{2} b_{3} \vee \bar{v} b_{1}, \\
f_{3}^{B}=\left(\bar{v} \vee b_{3}\right) \overline{b_{1} b_{2}} \vee v \overline{b_{1}} b_{2} \vee b_{1} b_{2} b_{3} \vee v b_{1} \overline{b_{2}} \overline{b_{3} .} .
\end{array}\right.
$$

and the output functions $h^{A}(a)=a_{3}$ and $h^{B}(b)=b_{3}$. The transition graphs of $\Sigma^{A}$ and $\Sigma^{B}$ and their semiattractors are represented in Fig. 4. The transition graph $G$ of the interconnected network has $2^{6}=64$ states. 
(a)

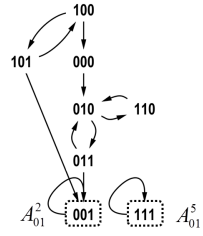

(c)

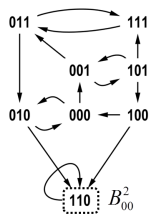

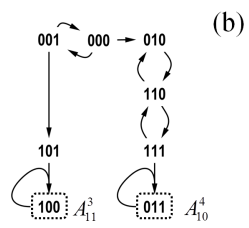

(b)

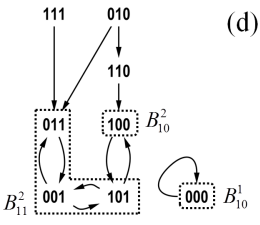

Figure 4: The respective transition graphs of $\Sigma^{A}$ and $\Sigma^{B}$ : (a) $G^{A, 0}$, (b) $G^{A, 1}$, (c) $G^{B, 0}$, (d) $G^{B, 1}$ (semi-attractors are framed).

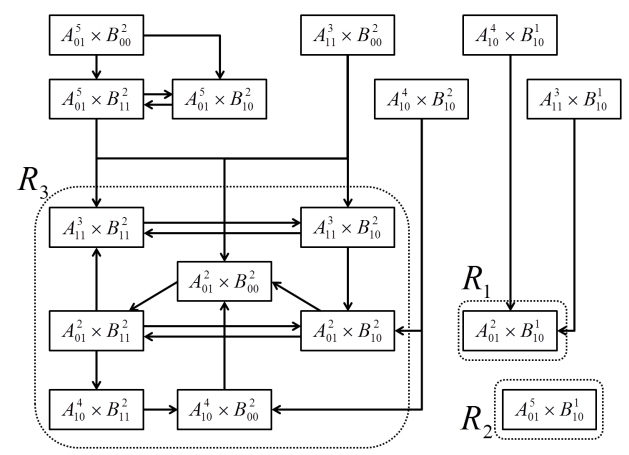

Figure 5: The asymptotic graph $\mathcal{G}^{\text {as }}$ of $\Sigma$ (self-loops are omitted).

This graph has two attractors, which are the steady states $Q_{1}=\{001000\}$ and $Q_{2}=\{111000\}$. The asymptotic graph $\mathcal{G}^{\text {as }}$ of the interconnection has only 16 vertices (which is a significant reduction with respect to $G$ ) and has three terminal SCCs: $R_{1}=A_{01}^{2} \times B_{10}^{1}, R_{2}=A_{01}^{5} \times B_{10}^{1}$ and $R_{3}$ contains 7 vertices (see Fig. 5). $R_{1}$ and $R_{2}$ predict the two attractors $Q_{1}$ and $Q_{2}$ of $G$ (Theorem 1), and $R_{3}$ is a spurious attractor. To see this, take the state 110011 , lying in $A_{10}^{4} \times B_{11}^{2} \in R_{3}$, as an initial state and apply the two following steps. First, fix coordinate $a$, and let $b$ evolve in $G^{B, 0}$. You obtain the path $110011 \triangleright_{G} 110000$ in $G$. Then, fix coordinate $b$, and let $a$ evolve in $G^{A, 0}$. You obtain the path $110000 \triangleright_{G} 001000$. Therefore, there is a path $110011 \triangleright_{G} 001000=Q_{1}$, and $Q_{1}$ is a steady state, so 110011 cannot belong to an attractor of $G$. This path is "hidden" in $\mathcal{G}^{\text {as }}$, as it is not allowed by Definition 4. More precisely, Step 1 stops $b$ at 000 which is transient in $G^{B, 0}$ (it does not belong to any attractor of $\Sigma^{B}$ ). This example explains how spurious attractors may appear in $\mathcal{G}^{\text {as }}$. It also explains why, on the contrary, there is a one-to-one correspondence in Theorem 2 . Indeed, in $\mathcal{G}^{\text {as }}$ the "switches" between $\Sigma^{A}$ and $\Sigma^{B}$ can occur only when terminal states are reached, whereas in $\mathcal{G}^{\text {cr }}$, the switches can occur at terminal and transient states. In other words, all paths in $G$ have a counterpart in $\mathcal{G}^{\text {cr }}$, but it is not the case in $\mathcal{G}^{\text {as }}$.

\section{B Proof of Proposition 1}

We give here the proof of Proposition 1, allowing to eliminate spurious attractors in some cases.

Proof. case i) Suppose $R=\{V\}$, with $V=A_{u \alpha}^{i} \times B_{v \beta}^{j}$. Since $R$ is a terminal SCC, all successors of $V$ must be equal to $V$, implying $V=A_{\beta \alpha}^{i} \times B_{\alpha \beta}^{j}$. It is easy to see that the set $\pi(R)=\pi(V)$ is then strongly connected in $G$ : take two elements $x=(a, b)$ and $x^{\prime}=\left(a^{\prime}, b^{\prime}\right)$ in $\pi(V)$. Since $a \sim_{G^{A, \beta}} a^{\prime}$ (they belong to the same SCC $\left.A_{\beta}^{i}\right)$ and $h^{B}(b)=\beta$, Lemma 2 implies $(a, b) \sim_{G}\left(a^{\prime}, b\right)$. In a similar way, $\left(a^{\prime}, b\right) \sim_{G}\left(a^{\prime}, b^{\prime}\right)$, yielding $x \sim_{G} x^{\prime}$. Now we prove that $\pi(R)$ is also an invariant of $G$. Let $x=(a, b) \in \pi(R)$ and consider any successor $x^{\prime}$ of $x$, for instance: $x^{\prime}=\left(a^{\prime}, b\right)$ with $a \rightarrow_{G^{A, \beta}} a^{\prime}$. Since $A_{\beta}^{i}$ is a terminal SCC of $G^{A, \beta}, a^{\prime}$ belongs to $A_{\beta \alpha^{\prime}}^{i}$, for some $\alpha^{\prime}$. If $\alpha^{\prime} \neq \alpha$, then $V$ would have a successor $V^{\prime} \neq V$. Therefore, $\alpha^{\prime}=\alpha$ and $x^{\prime} \in \pi(V)$. In conclusion, the set 
$\pi(R)$ is both strongly connected and invariant, which means that it is an attractor of $\Sigma$.

case ii) Suppose the $A$-output of $R$ is a singleton $\{\alpha\}$, then any $V$ in $R$ is of the form: $V=A_{(.) \alpha}^{(.)} \times B_{\alpha(.)}^{j}$, for some $j$. In other words, each $\pi^{B}(V)$ is a semi-SCC of the same terminal SCC $B_{\alpha}^{j}$ (since there are no paths between two distint terminal SCC). Suppose now that the set $S:=\{a: \exists b,(a, b) \in \pi(R)\}$ is an attractor of $G^{A, u}$ for all $u$. Then it is easy to see that $\pi(R)$ is strongly connected: if $(a, b)$ and $\left(a^{\prime}, b^{\prime}\right)$ are two elements of $\pi(R)$, then $(a, b) \sim_{G}\left(a^{\prime}, b\right)$ (since $S$ is an attractor of $\left.G^{A, h^{B}(b)}\right)$ and $\left(a^{\prime}, b\right) \sim_{G}\left(a^{\prime}, b^{\prime}\right)$ (since $B_{\alpha}^{j}$ is an attractor of $\left.G^{B, \alpha}\right)$. To show that $\pi(R)$ contains all its successors, suppose first that $(a, b) \rightarrow_{G}\left(a^{\prime}, b\right)$ : then $a^{\prime} \in S(S$ is an attractor of $G^{A, h^{B}(b)}$ ) which implies that there exists $b^{\prime}$ such that $\left(a^{\prime}, b^{\prime}\right) \in \pi(R)$. Therefore, we have $\pi(R) \ni(a, b) \rightarrow_{G}\left(a^{\prime}, b\right) \sim_{G}\left(a^{\prime}, b^{\prime}\right) \in \pi(R)$, which gives $\left(a^{\prime}, b\right) \in \pi(R)$. Suppose next that $(a, b) \rightarrow_{G}\left(a, b^{\prime}\right)$ : $b^{\prime}$ necessarily belongs to the terminal SCC $B_{\alpha}^{j}$, and $\left(a, b^{\prime}\right) \in \pi(R)$. Again, $\pi(R)$ is both strongly connected and invariant, so it is equal to an attractor of $\Sigma$.

case iii) is analogous to case ii).

\section{Boolean model of the segment polarity network}

A Boolean version of the segment polarity network was proposed in [2] (see also [6, 7]). It is composed of a four cells segment, with periodic boundary conditions. Each cell receives two inputs (proteins WG wingless and $\mathrm{HH}$ hedgehog) from its left and right neighbors. With respect to [2], the model has been simplified to consider only the proteins (whenever mRNA' $=F(X)$ and $\mathrm{P}^{\prime}=$ mRNA, we set $\mathrm{P}^{\prime} \equiv F(X)$ ). There are two types of cellular modules, depending on their (constant) concentration of $\operatorname{SLP} \in\{0,1\}$. The segment polarity genes interactions for one cell and the interconnection scheme of the segment are represented in Figure 6.

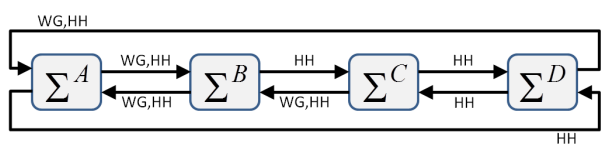

\begin{tabular}{|c|c|c|c|}
\hline \multicolumn{3}{|c|}{$\begin{array}{l}\text { Inputs: } u=\left(u_{1}, u_{2}\right) \\
\qquad u=\left(u_{1}, u_{2}, u_{3}, u_{4}\right) \\
\text { Outputs: } h_{1}=\mathrm{HH}, h_{2}=\mathrm{WG} \\
\text { Logical rules: }\end{array}$} & $\begin{array}{l}\text { (cell of type SLP=1) } \\
\text { (cell of type } S L P=0)\end{array}$ \\
\hline WG' & $=$ & $(\mathrm{CIA} \vee \mathrm{WG}) \overline{\mathrm{CIR}}$ & (cell of type SLP $=1$ ) \\
\hline EN' & $=$ & 0 & (cell of type SLP=1) \\
\hline WG' & $=$ & WG CIA $\overline{\text { CIR }}$ & e $\mathrm{SLP}=0$ ) \\
\hline EN' & $=$ & $u_{3} \underline{\vee u_{4}}$ & (cell of type SLP=0) \\
\hline $\mathrm{HH}^{\prime}$ & $=$ & EN $\overline{\mathrm{CIR}}$ & \\
\hline PTC' & $=$ & $(\mathrm{CIA} \overline{\mathrm{EN}} \overline{\mathrm{CIR}}) \vee(\mathrm{H}$ & $\left.\overline{t_{1}} \overline{u_{2}}\right)$ \\
\hline $\mathrm{CI}^{\prime}$ & $=$ & $\overline{\mathrm{EN}}$ & \\
\hline CIA' & $=$ & $\mathrm{CI}\left(\overline{\mathrm{PTC}} \vee u_{1} \vee\right.$ & \\
\hline CIR' & $=$ & CI PTC $\overline{u_{1}} \overline{u_{2}}$ & \\
\hline
\end{tabular}

Figure 6: Segment and modules definitions of segment polarity network [2]. 